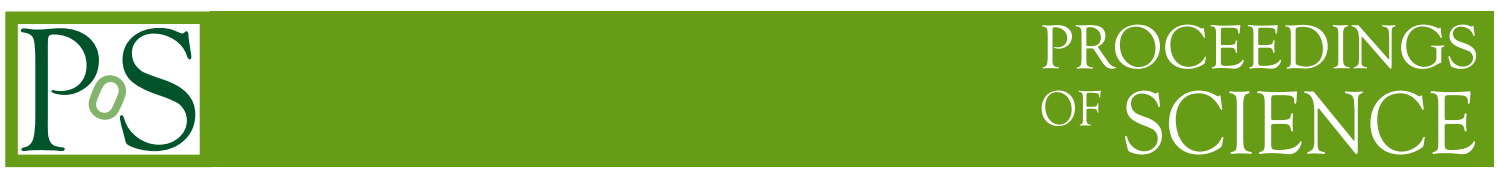

\title{
Quantum Nambu Geometry in String Theory
}

\section{Chong-Sun Chu*i}

Centre for Particle Theory and Department of Mathematical Sciences,

Durham University, Durham, DHI 3LE, UK

E-mail: chong-sun. chu@durham.ac.uk

Proceedings of the Corfu Summer Institute 2011 School and Workshops on Elementary Particle Physics and Gravity

September 4-18, 2011

Corfu, Greece

* Speaker.

${ }^{\dagger}$ A footnote may follow. 


\section{Introduction}

It is generally expected that the usual description of spacetime in terms of Riemannian geometry would break down above the Planck energy scale. A possibility is that geometry is quantized and spacetime coordinates become quantum operators. In this case, traditional spacetime concepts such as locality and causality, and even the fundamental nature of spacetime itself, will have to be re-examined. String theory, as a candidate for a theory of quantum gravity, provides an interesting setup to address some of these questions.

In the paper [1], a new kind of quantized geometry was discovered. It is characterized by the quantum Nambu bracket

$$
[f, g, h]:=f g h+g h f+h f g-f h g-g f h-h g f,
$$

where $f, g, h$ are any three operators and the binary product is the usual operator product. This differs from the previous examples of noncommutative geometry in string theory which are characterized by a commutator and could be referred to as of Lie-algebra type.

For the standard Lie algebraic type noncommutative geometry [2-7], a 2-form field strength can be written as

$$
F^{\mu v}=-i\left[X^{\mu}, X^{v}\right]
$$

when the fluctuation over the noncommutative geometry background is taken into account. What about the fluctuation around the quantum Nambu geometry? It is suggestive to interpret the quantum Nambu bracket of the target space coordinate fields $X^{\mu}$ as a 3-form field strength

$$
H^{\mu \nu \lambda}=-i\left[X^{\mu}, X^{v}, X^{\lambda}\right] .
$$

To check this idea, we have to look for a place where a non-abelian 3-form field strength lives. This leads us to consider the system of multiple D4-branes (where the 3-form field strength would be the Hodge dual of a 2-form field strength) and multiple M5-branes (where the 3-form field strength would be self-dual).

To reach the D4-branes system, we use the D1-strings matrix model to derive the Matrix model descriptions for the II B string, II A string and M-theory in a corresponding background of large RR-flux or its uplift to eleven dimensions. For the II A Matrix string theory, we find that a classical solution of quantum Nambu geometry is again allowed. We also find that the fluctuation around the solution gives a Lagrangian for a 1-form gauge potential whose form is exactly the same as the dimensional reduced PST action (which describes a single D4-brane) [8] if a quantum Nambu bracket of $X^{\mu}$ is identified as (1.3) as a 3-form field strength whose Hodge dual would be the Yang-Mills field strength. Physically, this means the system of fundamental strings has expanded over the quantum Nambu geometry into a system of multiple D4-branes.

Since a system of multiple D4-branes can be considered as a dimensional reduction of multiple M5-branes on a circle, it has been proposed recently that $[9,10]$ the instantons on the D4-branes can be identified with the KK modes of the compactified M5-branes, and that by including all the instantons, the D4-branes SYM theory is in fact equal to the M5-branes theory. In view of this, we suggest a natural way to include all the KK modes into the D4-branes and propose the action (4.4) for multiple 
M5-branes in a constant self-dual $C$-field. Our proposed action is living on a 6-dimensional quantum Nambu geometry with self-dual parameter $\theta^{\mu \nu \lambda}$ and is formulated in terms of a non-abelian 3-form field strength defined using (1.3). A priori, such an $H^{\mu \nu \lambda}$ may not obey the desired self-duality condition. Nevertheless quite amazingly we find that the self-duality condition emerges naturally from our model. The M5-branes system in a $C$-field could be reduced to a system of D4-branes in $B$-field, and the latter has a worldvolume described by the standard Moyal type noncommutative geometry. This connection allows us to identify the $\theta^{\mu \nu \lambda}$ parameter of the quantum Nambu geometry as a $C$-field on the worldvolume of the M5-branes. Therefore we obtain the result that the worldvolume of the M5-branes in a $C$-field is described by a quantum Nambu geometry

$$
\left[X^{\mu}, X^{v}, X^{\lambda}\right]=i \theta^{\mu \nu \lambda}
$$

with self-dual parameters $\theta^{\mu \nu \lambda}=C^{\mu \nu \lambda}$.

\section{Matrix Model of D1-Strings in Large RR 3-Form Flux}

We start with the supergravity background of [11]: The metric (in the string frame) takes the form of $\mathbb{R}^{3} \times A d S_{2} \times S^{5}$ :

$$
d s^{2}=\sum_{i=1}^{3}\left(d X^{i}\right)^{2}+R^{2}\left(\frac{-d t^{2}+d U^{2}}{U^{2}}\right)+R^{\prime 2} d \Omega_{5}^{2}
$$

where

$$
R^{2}=2 e^{-2 \Phi} / f^{2}, \quad R^{\prime 2}=80 e^{-2 \Phi} / f^{2},
$$

and $d \Omega_{5}^{2}=\hat{G}_{i^{\prime} j^{\prime}} d X^{i^{\prime}} d X^{j^{\prime}}$ is the metric for an $S^{5}$ of unit radius. In addition, there is a nonvanishing dilaton, axion, RR potentials $C_{2}$ and $C_{4}$ specified by:

$$
\begin{aligned}
e^{-\Phi} & =\chi /(2 \sqrt{2})=\text { constant }, \\
F_{3} & = \begin{cases}f \varepsilon_{i j k}, & i, j, k=1,2,3, \\
0, & \text { otherwise },\end{cases} \\
F_{5} & = \begin{cases}c \varepsilon_{5} & \text { on } \mathscr{M}_{5}, \\
c \varepsilon_{5}^{\prime} & \text { on } \mathscr{M}_{5}^{\prime}, \\
0 & \text { otherwise }\end{cases}
\end{aligned}
$$

and $f^{2}=\frac{2}{3} c^{2}$.

Next let us consider a system of $N$ parallel D1-branes in this background. The worldvolume action for the D1-branes is given by

$$
S_{D 1}:=S_{X}+S_{C S}+S_{Y M},
$$

where $S_{C S}$ is the Chern-Simons term of the Myers type given by

$$
\begin{aligned}
S_{C S} & =\mu_{1} \int \operatorname{Tr}\left[\lambda F \chi+P C_{2}+i \lambda^{2} F \mathrm{i}_{\Phi} \mathrm{i}_{\Phi} C_{2}+i \lambda P \mathrm{i}_{\Phi} \mathrm{i}_{\Phi} C_{4}-\frac{\lambda^{3}}{2} F \mathrm{i}_{\Phi}^{4} C_{4}\right] \\
& :=S_{\chi}+S_{C_{2}}+S_{C_{4}} .
\end{aligned}
$$


It was shown in [1] that there is a low energy large flux double scaling limit so that the term $S_{C_{2}}$ dominates and one can ignore all the other terms in the action, In this limit, the system of D1-branes is govern by

$$
\begin{aligned}
S_{C_{2}} / \mu_{1} & =f \int d^{2} \sigma \operatorname{Tr}\left(\frac{1}{2} \varepsilon_{i j k} X^{i} D_{\alpha} X^{j} D_{\beta} X^{k} \varepsilon^{\alpha \beta}\right)+f \int d^{2} \sigma \operatorname{Tr}\left(i F X^{i} X^{j} X^{k} \varepsilon_{i j k}\right) \\
& \equiv f \int d^{2} \sigma\left(L_{1}+L_{2}\right) .
\end{aligned}
$$

The classical solution to the equation of motion was obtained in [1]. It was found a new solution

$$
\left[X^{i}, X^{j}, X^{k}\right]=i \theta \varepsilon^{i j k}
$$

where $\theta$ is a constant and the 3 -bracket is given by quantum Nambu bracket.

It was also shown that in the large $N$ limit, there are new representations of (2.10) that are not given by Lie algebraic representation, The existence of these representations that demonstrates the fundamental and novel nature of the Nambu-Heisenberg commutation relation (2.10). In other words, a quantized geometry, the quantum Nambu geometry, characterized by the Nambu bracket (1.1) is allowed as a solution in string theory.

\section{D4-branes in Large RR Flux Background}

We would like to perform an expansion around the quantum Nambu geometry and ask what kind of gauge theory would come out. We recall that for the standard Lie algebraic type noncommutative geometry, a 2-form field strength is obtained from the fluctuation over the noncommutative geometry as

$$
F^{\mu v}=-i\left[X^{\mu}, X^{v}\right]
$$

For our quantum Nambu geometry, it is suggestive to interpret the quantum Nambu bracket of the target space coordinate fields $X^{\mu}$ as a 3-form field strength

$$
H^{\mu v \lambda}=-i\left[X^{\mu}, X^{v}, X^{\lambda}\right]
$$

and we would like to check this idea.

Places where a non-abelian 3-form field strength lives are, for example, multiple D4-branes (where the 3-form field strength would be the Hodge dual to a 2-form field strength) and multiple M5-branes (where the 3-form field strength would be self-dual). To check the idea, we would like to connect to these systems from our D1-branes system. And to do this, let us first derive the Matrix model descriptions for the IIB string theory, M-theory and II A string theory in a large flux background using our description (2.9) for the D1-branes.

In [1], a duality argument was performed and a Matrix IIA string theory that is dual to the above D1-string matrix theory was obtained. The action reads

$$
S_{I I A}=\frac{f}{N} \int d^{2} \sigma \operatorname{Tr} X^{a} X^{b} X^{c} X^{d} X^{e} \varepsilon_{a b c d e}, \quad a, b, c, d, e=0,1,2,3,4,
$$


where

$$
X^{\alpha}=i D^{\alpha}, \quad X^{i}=\text { scalars }, \quad \alpha=0,1, \quad i=2,3,4
$$

We note that the D1-strings action and the II A Matrix string action are indeed the same up to a constant coefficient. This is similar to the matrix IIA string of DVV where the same 2-dimensional supersymmetric Yang-Mils theory could have different string interpretations depending on how one associate its parameters with the string theories. This is consistent with T-duality.

The matrix string action (3.3) admits the classical solution:

$$
\left[X^{\alpha}, X^{\beta}\right]=0, \quad\left[X^{\alpha}, X^{i}\right]=0, \quad \alpha=0,1, i=2,3,4 .
$$

As before, the commutation relations of $X^{i}$ among themselves are not constrained. Let us consider the solution $X_{c l}^{i}=x^{i}$ of quantum Nambu geometry

$$
\left[x^{2}, x^{3}, x^{4}\right]=i \theta
$$

and consider a fluctuation around it. In the large $N$ limit, we get a set of large $N$ matrices $x^{i}$. Depending on the representation chosen, they may or may not generate the entire set of $N \times N$ matrices. In general, assume $x^{i}$ do not generate the whole set of $N \times N$ matrices. Then every $N \times N$ matrix can be expressed as a $K \times K$ matrix whose entries are functions of $x^{i}$. The expansion of the dynamical variables around the classical solution can thus be parameterized as

$$
X^{i}=x^{i} \mathbf{1}_{K \times K}+A^{i}\left(\sigma, x^{j}\right) .
$$

The action (3.3) becomes

$$
S_{5}=\frac{f}{N} \int_{\Sigma_{5}} \operatorname{tr} X^{a} X^{b} X^{c} X^{d} X^{e} \varepsilon_{a b c d e}
$$

where $\int_{\Sigma_{5}}=\int d^{2} \sigma \int_{x}$ and $\int_{x}$ is an integral on the quantum Nambu geometry which can be constructed from a representation of the geometry. In the large $N$ limit, the trace over large $N$ matrices decompose as usual as $\operatorname{Tr}=\int_{x}$ tr.

With the introduction of a three-form $H$-field whose components are defined by

$$
\begin{aligned}
& H^{a b c}=-i\left[X^{a}, X^{b}, X^{c}\right], \\
& H^{d e 5}=-i\left[X^{d}, X^{e}\right], \quad a, b, c, d, e=0,1,2,3,4,
\end{aligned}
$$

where

$$
H^{* \mu \nu \lambda}:=\frac{1}{6 \sqrt{-g}} \varepsilon^{\mu \nu \lambda \rho \alpha \beta} H_{\rho \alpha \beta}
$$

is the Hodge dual of $H_{\rho \alpha \beta}$, the action (3.8) becomes

$$
S_{5}=\int_{\Sigma_{5}} \operatorname{tr} H^{a b c} H^{d e 5} \varepsilon_{a b c d e}
$$

In [1], it was argued that this matches with the dimension reduced PST action and so describes a sector of multiple D4-branes theory (where $X^{a^{\prime}}=0$ ) in a large RR 2-form flux background. 


\section{A proposal for a theory of multiple M5-branes using 1-form gauge field}

Recently, it has been argued that $[9,10]$ the instantons on multiple D4-branes could be identified with the KK modes associated with the compactification of M5-branes on a circle. By including all these modes, it was proposed that the low energy SYM theory of D4-branes is a well-defined quantum theory and is actually the theory of multiple M5-branes compactified on a circle. Back to our proposed action (3.12) for D4-branes in a large RR flux background, how can we incorporate the higher KK modes in our description? A possible hint is from the identification (3.10). We note that the identification for $H^{d e 5}$ can be written as

$$
H^{d e 5}=-i\left[X^{d}, X^{e}, X^{5}\right]
$$

with

$$
X^{5}=1 .
$$

If we think about $X^{5}$ as a scalar field describing the compactified $X^{5}$ direction transverse to the D4brane, then one can understand the relation (3.10) and (4.2) as saying only the zero mode of the M5branes has been included, i.e. a dimensional reduction to D4-branes. In this picture, it is suggestive to include the higher KK/instantonic modes by promoting $X^{5}=\mathbf{1}$ to a general field. The identification (3.9) and (4.1) can be put together as

$$
H^{\mu \nu \lambda}=-i\left[X^{\mu}, X^{v}, X^{\lambda}\right]
$$

It was proposed in [1] to be a different way to write the non-abelian self-dual 3-form field strength living on M5-branes. In a conventional description, there would be a non-abelian 2-form potential $B$ and $H=d B+\cdots$ where the $\cdots$ term denotes terms necessarily for the non-abelianization. Thus we are proposing that there is a dual description of the non-abelian 3-form field strength in terms of the 1-form variables $X=X_{\mu} d \sigma^{\mu}$; and the $B$-field and the $X$-field are related, although one can expect the relation to be very complicated. To justify our proposal, one needs to show that $H^{\mu \nu \lambda}$ satisfies the correct equation of motion (i.e. the self-dual equation) and describes the correct number of on-shell degrees of freedom (i.e. three). In [1], the following action

$$
S_{M 5, \theta}=-\frac{1}{4} \int_{x} \operatorname{tr}\left(\frac{1}{6} \varepsilon_{a b c d e} H^{a b c} H^{d e 5}+\left(\alpha \frac{-1}{3} H^{a b c} H_{a b c}+(1-\alpha) H^{a b 5} H_{a b 5}\right) \sqrt{-g}\right),
$$

$(\alpha=1 / 6)$ was proposed. The theory has solution

$$
\left[x^{\mu}, x^{v}, x^{\lambda}\right]=i \theta^{\mu \nu \lambda} \mathbf{1},
$$

where $\theta^{\mu \nu \lambda}$ are arbitrary self-dual constants. This corresponds to a six dimensional quantum Nambu geometry parameterized by self-dual parameter $\theta^{\mu \nu \lambda}$ and the trace $\int_{x}$ is determined from the representations of the quantum Nambu geometry (4.5)

The fluctuations around the solution can be written as

$$
X^{\mu}=x^{\mu} \mathbf{1}_{K \times K}+A^{\mu}(x),
$$

where $A^{\mu}$ are $K \times K$ matrices. The theory (4.4) over the geometry (4.5) was proposed to describe a theory of $K$ M5-branes. 
We note that our M5-branes system has a quantum Nambu geometry as its worldvolume geometry. What is the physical origin responsible for this quantized spacetime? The emergence of a noncommutative worldvolume on a brane is typically the result of a background gauge potential being turned on in its worldvolume. The fact that the quantization parameter $\theta^{\mu \nu \lambda}$ is self-dual suggests to identify it with the self-dual 3-form $C$-field on the worldvolume of the M5-branes. This identification is further supported by the fact that if we dimensionally reduced the M5-branes, say, on the 5-th direction, which amounts to putting $X^{5}=\mathbf{1}$, then the relation (4.5) reads

$$
\left[X^{\mu}, X^{v}, \mathbf{1}\right]=\left[X^{\mu}, X^{v}\right]=i \theta^{\mu v 5} .
$$

This is the noncommutative geometry over D4-branes with a $B$-field whose components are $B_{\mu \nu}=\theta_{\mu v 5}$ (we remind the readers we are considering the linearized limit). Since the $B$-field is related to the 11dimensional $C$-field as $B_{\mu \nu}=C_{\mu \nu 5}$, it is correct to identify $\theta^{\mu \nu \lambda}$ with the constant $C$-field $C^{\mu \nu \lambda}$. All in all, we conclude that the geometry (4.5) is the result of having a self-dual 3 -form $C$-field

$$
C_{\mu v \lambda}=\theta_{\mu \nu \lambda}
$$

turned on in the worldvolume of the M5-branes.

In our description, the field strength $H^{\mu \nu \lambda}$ is constructed from the 1-form potentials $A^{\mu}$ using (4.3) and (4.6). In a conventional description of 3-form field strength, a 2-form potential is used. Our analysis suggests that there maybe in fact two equivalent formulations for the theory of multiple M5-branes in a $C$-field, one in terms of a 1-form gauge field as in ours (4.4), and the conventional formulation in terms of a 2 -form gauge potential.

Evidence of this can be seen from the counting of the degrees of freedom of our model. Initially we have six fields. If the self-duality equation is in fact the equation of motion of the theory, then the degrees of freedom are reduced to half and we have indeed three degrees of freedom which is appropriate for a description of a self-dual 3-form field strength. The theory (4.4) would then have all the desirable properties of a theory of non-abelian self-dual 3-form field strength except that the theory is written manifestly using a 1-form potential as the variables.

A couple of comments on the dual formulation are in order:

1. As noted above, our action (4.4) is equal to the non-abelian form of the PST action when the self-duality condition is satisfied. The agreement of the actions on-shell is a necessary condition for our formulation to be an equivalent description on-shell. Therefore this agreement provides more support that our proposed action (4.4) indeed provides a dual description of the non-abelian self-dual 3-form.

2. Our formulation of using a 1-form gauge field $A_{\mu}$ is supposed to be equivalent to the conventional formulation of using a 2 -form gauge potential $B_{\mu \nu}$ only on-shell. As such there can be a relation between the 2-form gauge field and the 1-form gauge field only on-shell. Identifying such a $B$-field from our description is important as it would allow us to couple to self-dual strings.

3. In the conventional formulation, the existence of the tensor gauge symmetry and the self-duality equation is crucial in reducing the fifteen components of $B$ to three. In our formulation, we do 
get the desired number of degree of freedom (modulo the issues discussed above) and there is no need of a tensor gauge symmetry. Curiously, in a recent construction of the non-abelian 3-form theory using a 2-form $B$-field potential [13], it was shown that the tensor gauge symmetry (part of the $G \times G$ symmetry structure constructed there) could be gauge fixed to an ordinary gauge symmetry $G$ (diagonal part of $G \times G$ ). It is interesting that the gauge fixed theory has precisely the same gauge symmetry as our proposed description here. This coincidence provides some support to both the description proposed in [13] and the description proposed here.

4. In the above we have obtained the Bianchi identity and the self-duality condition as a solution of the reduced matrix description. However to fully justify our proposal, we need to establish that it is the only nontrivial solution. We recall that in the PST action, one does not get the self-duality condition as the equation of motion immediately. To do this, one needs to make crucial use of a symmetry which acts on the $B$-field. For our case, it is possible that there is a counterpart of the symmetry which acts on the $X$ 's; and this symmetry is needed to derive the self-duality equation (and hence the Bianchi identity). It is important to understand whether such a symmetry really exists in our model, and if so, how it acts.

Another possible way to settle the issue is to supersymmetrize our action with $(1,0)$ or $(2,0)$ supersymmetry since supersymmetry would require the 3-form field strength to be self-dual automatically. Supersymmetrization of our system is also needed for describing M5-branes. In any case supersymmetry is an important topic and we hope to return to it in future work. See [12] for some recent related works on $(2,0)$ supersymmetry of a non-abelian self-dual 3-form field strength multiplet.

More recently, an non-abelian action for multiple M5-branes was constructed [14]. In this formulation, the tensor symmetry is abelian but the theory carry with it a $U(N)$ Yang-Mills gauge symmetry. It is not clear yet the connection between these different formulations, but it is encouraging to see the same gauge group emerges.

\section{References}

[1] C. -S. Chu and G. S. Sehmbi, "D1-Strings in Large RR 3-Form Flux, Quantum Nambu Geometry and M5-Branes in C-Field,' J. Phys. A A 45 (2012) 055401 [arXiv:1110.2687 [hep-th]].

[2] M. R. Douglas and C. M. Hull, "D-branes and the noncommutative torus," JHEP 9802 (1998) 008 [arXiv:hep-th/9711165].

[3] C. S. Chu and P. M. Ho, "Noncommutative open string and D-brane," Nucl. Phys. B 550 (1999) 151 [arXiv:hep-th/9812219].

C. S. Chu and P. M. Ho, "Constrained quantization of open string in background B field and noncommutative D-brane," Nucl. Phys. B 568 (2000) 447 [arXiv:hep-th/9906192].

C. S. Chu, "Noncommutative open string: Neutral and charged," arXiv:hep-th/0001144.

[4] V. Schomerus, "D-branes and deformation quantization," JHEP 9906 (1999) 030 [arXiv:hep-th/9903205].

[5] N. Seiberg and E. Witten, "String theory and noncommutative geometry," JHEP 9909 (1999) 032 [arXiv:hep-th/9908142]. 
[6] A. Y. .Alekseev, A. Recknagel, V. Schomerus, "Noncommutative world volume geometries: Branes on SU(2) and fuzzy spheres," JHEP 9909 (1999) 023. [hep-th/9908040].

[7] A. Y. .Alekseev, A. Recknagel, V. Schomerus, "Brane dynamics in background fluxes and noncommutative geometry," JHEP 0005 (2000) 010. [hep-th/0003187].

[8] P. Pasti, D. P. Sorokin, M. Tonin, "Covariant action for a D = 11 five-brane with the chiral field," Phys. Lett. B398 (1997) 41-46. [hep-th/9701037].

[9] M. R. Douglas, “On D=5 super Yang-Mills theory and (2,0) theory,” JHEP 1102 (2011) 011. [arXiv:1012.2880 [hep-th]].

[10] N. Lambert, C. Papageorgakis, M. Schmidt-Sommerfeld, "M5-Branes, D4-Branes and Quantum 5D super-Yang-Mills,” JHEP 1101 (2011) 083. [arXiv:1012.2882 [hep-th]].

[11] C. -S. Chu, P. -M. Ho, "D1-brane in Constant R-R 3-form Flux and Nambu Dynamics in String Theory," JHEP 1102 (2011) 020. [arXiv:1011.3765 [hep-th]].

[12] N. Lambert, C. Papageorgakis, “Nonabelian (2,0) Tensor Multiplets and 3-algebras,” JHEP 1008 (2010) 083. [arXiv:1007.2982 [hep-th]].

N. Lambert, P. Richmond, “(2,0) Supersymmetry and the Light-Cone Description of M5-branes,” [arXiv:1109.6454 [hep-th]].

C. Papageorgakis, C. Saemann, "The 3-Lie Algebra (2,0) Tensor Multiplet and Equations of Motion on Loop Space,” JHEP 1105 (2011) 099. [arXiv:1103.6192 [hep-th]].

[13] C. -S. Chu, "A Theory of Non-Abelian Tensor Gauge Field with Non-Abelian Gauge Symmetry G x G," arXiv:1108.5131 [hep-th].

[14] C. -S. Chu and S. -L. Ko, "Non-abelian Action for Multiple M5-Branes," arXiv:1203.4224 [hep-th]. 